\title{
Incomodidad Corporal, Carga Física y Nivel de Flexibilidad en Trabajadores del Sector Administrativo de una Institución de Enseñanza Superior en Florianópolis, del Sur de Brasil
}

\section{BODY DISCOMFORT, CARGO PHYSICAL AND FLEXIBILITY IN LEVEL WORKERS IN THE ADMINISTRATIVE SECTOR A HIGHER EDUCATION INSTITUTION IN SOUTHERN BRAZIL}

Ines Alessandra Xavier Lima', Luana Meneghini Belmonte², Antonio Renato Pereira Moro ${ }^{3}$, Armando Monterrosa Quintero ${ }^{4}$

1. Estudiante de Posgrado en Ingeniería de Producción, Universidad Federal de Santa Catarina.

2. Profesora Universidad del Sur de Santa Catarina (Brasil).

3. Profesor Programa de Posgrado en Ingeniería de Producción, Universidad Federal de Santa Catarina (Brasil).

4. Estudiante de Posgrado en Biodinámica del Desempeño Humano, Universidad Federal De Santa Catarina (Brasil).

\section{RESUMEN}

El objetivo de este estudio fue identificar el perfil de los funcionarios de una oficina de una institución de enseñanza superior con relación a las tareas desarrolladas y a sus manifestaciones de dolor, incomodidad corporal. Hicieron parte de este estudio 41 funcionarios que desempeñan sus actividades laborales en posición sentada. Los instrumentos utilizados en la colecta de datos fueron: la escala de Dolor o incomodidad corporal, o método RULA y el banco de Wells. Los datos fueron analizados por medio de estadística descriptiva (media, desviación estándar, frecuencia relativa) y estadística inferencial. En cuanto al análisis de las posiciones de los diferentes segmentos se observó que ninguna posición fue considerada aceptable; en pocas palabras, todas las posturas generan un riesgo a la salud del trabajador. Consideramos de esta manera un perfil negativo de los funcionarios del presente estudio con relación a la presencia de manifestación de dolor, incomodidad corporal, posiciones y sobrecarga biomecánica durante la realización de tareas y nivel de flexibilidad corporal. Que separadamente o en conjunto causan riesgo a la salud del trabajador afectando posiblemente su desempeño y calidad de vida.

(Xavier I, Meneghini L, Pereira A, Monterrosa A, 2016. Incomodidad Corporal, Carga Física y Nivel de Flexibilidad en Trabajadores del Sector Administrativo de una Institución de Enseñanza Superior en Florianópolis, del Sur de Brasil. Cienc Trab. Sep-Dic; 18 [57]: 145-149).

Palabras claves: INGENIERÍA HUMANA, SALUD LABORAL, MODALIDADES DE POSICIÓN.

\section{ABSTRACT}

The aim of this study was to identify the profile of the employees of a Secretary of an Education Institution with regard to the complaint of pain and discomfort body. Participants were 41 employees who perform their work activities in sitting on computerized terminals. The instruments used in data collection were: Scale of Pain and/or Discomfort Body, RULA method, Wells's seat. The data were processed using descriptive statistics (mean, standard deviation, relative frequency) and inferential. The analysis of the attitudes of different segments was observed that no position was considered acceptable, it means, all postures generate risk on health of the worker. It is thus a negative profile of employees of this study with regard to the complaint of pain and discomfort body postures and biomechanical overload during task performance and level of body flexibility, which separately or together may cause risks to health worker possibly affecting their performance and quality of life.

Keywords: HUMAN ENGINEERING, OCCUPATIONAL HEALTH, MODALITIES POSITION

\section{INTRODUCCIÓN}

En la actualidad, los sintomas resultantes de los acontecimientos osteo-musculares relacionados con las actividades ocupacionales son considerados los factores más sobresalientes en las incapacidades de

\section{Correspondencia / Correspondence:}

Armando Monterrosa Quintero

Universidad Federal de Santa Catarina

Trindade, Caja Postal 476 CEP 88040-900

Florianópolis, Santa Catarina, Brasil

Tel.: 05548 9833-6029.

e-mail: adomonterrosa@hotmail.com

Recibido: 01 de Agosto de 2016 / Aceptado: 17 de Octubre de 2016 los trabajadores. Los mismos pueden generar dolor y incomodidad corporal y una consecuente repercusión en la salud general del trabajador. ${ }^{1,2}$ Se considera que el dolor es uno de los síntomas más mencionados, principalmente en los relacionados con la mala posición del cuerpo, que a su vez se refleja en la producción laboral. Teniendo en cuenta estos factores, su control es esencial tanto del punto vista de la salud del trabajador y su desempeño. ${ }^{3}$ La relación del dolor, incomodidad e incomodidad asociados a los factores de riesgo en el ambiente de trabajo, como las posiciones extremas, utilización de la fuerza de forma repetida y ausencia de descanso, puede ser entendida como una de las primeras indicaciones de la necesidad de las investigaciones. ${ }^{4}$ La investigación, análisis y estudio de los factores de riesgo presentes en los diversos ambientes ocupacionales son fundamentales para la planeación e implementación de intervenciones preventivas eficaces y la prevención parece ser una de las 
mejores maneras para evitar la aparición de los disturbios osteomusculares relacionados con el trabajo.,5 En el ambiente ocupacional, varios son los factores incluidos en el desarrollo de estos disturbios. Por lo tanto, algunos estudios apuntan como los principales componentes de riesgos biomecánicos como: la fuerza excesiva, repetición de tareas, velocidad de los movimientos, duración de las actividades, posiciones laborales no adecuadas y asimétricas, entre otras. ${ }^{6}$ Los trabajadores que laboran en puestos de tipo informático permanecen largos periodos de tiempo en posiciones que exigen trabajo estático de los músculos. Si son de carácter repetitivo, prolongados y excesivos, las exigencias de trabajo estático pueden establecer incomodidad y dolores mayores o menores, localizados no solo en la parte muscular sino también en la parte articulatoria, tendinosa y otros tejidos corporales. ${ }^{7}$ Debido a la necesidad de la presencia de equipo informático en los puestos de trabajo, de naturaleza administrativa y el aumento de problemas músculo-esqueléticos en los trabajadores que los utilizan ${ }^{5}$, es de vital importancia la evaluación de los puestos de trabajo, permitiendo una ejecución correcta del trabajo y preservando la salud y seguridad del trabajador. ${ }^{8,9}$ La posición sentada es una de las más utilizadas en los puestos de trabajo de tipo informático, por permitir al trabajador disminuir el gasto energético, obtener mayor estabilidad en determinadas tareas y reducir los esfuerzos en las articulaciones de los miembros inferiores. Esta situación puede provocar alteraciones músculo-esqueléticas desencadenando sensaciones de incomodidad y dolor, generando la evolución de dolores crónicos. ${ }^{10,11}$ El seguimiento a las manifestaciones de dolor y la incomodidad relacionada al sistema músculo-esquelético es una de las formas de evaluar la aparición de sintomas corporales que puede servir como alerta para la prevención de las enfermedades de tipo laboral (DORT). El acontecimiento del DORT puede limitar o disminuir la interacción del hombre con el sistema de trabajo. En pocas palabras, puestos de trabajo que presentan prevalencias significativas de los sintomas mencionados, especialmente cuando se encuentran presentes en varios trabajadores que realizan ocupaciones semejantes y que manifiesten sintomatología análoga, deben ser objeto de atención e intervención ergonómica. ${ }^{12}$ El objetivo principal de nuestro estudio es identificar la presencia de manifestaciones de dolor, incomodidad corporal y carga física durante la realización de tareas de flexibilidad corporal de los funcionarios de una oficina de la institución de enseñanza superior que trabajan en posición sentada.

\section{MATERIALES Y MÉTODOS}

La investigación es de tipo aplicada, de campo y descriptiva; fue realizada en 2012 en una oficina de una universidad de Florianópolis, Santa Catarina, Brasil, con 41 funcionarios que desempeñan sus funciones laborales en posición sentada en escritorios, siguiendo las siguientes etapas:

- Etapa 1. Presentación de la investigación al comité de ética: el presente trabajo siguió las recomendaciones de la resolución 196/96 del Consejo Nacional de Salud para estudios con seres humanos y fue aprobado por el Comité de Ética en investigación de la Universidad del Sur de Santa Catarina (UNISUL) bajo el código 11.488.4.08.III.

- Etapa 2. Al obtener la aprobación, el proyecto fue presentado a los funcionarios y se realizó la convocatoria para las personas que desean ser parte de la investigación. Bajo un acuerdo firmaron el consentimiento informado y fue definido el día y horario para la recolección de datos teniendo en cuenta la disponibilidad en el horario de trabajo.

- Etapa 3. Recolección de datos: el registro de la información fue realizado en los puestos de trabajo de los participantes, utilizando los instrumentos y procedimientos descritos a seguir.

Los instrumentos utilizados en la recolección de la información fueron: Nivel de incomodidad para las diferentes partes del cuerpo $^{13}$, el método RULA ${ }^{14}$ y el banco de Wells para la realización del test "sentarse y alcanzar"15".

La escala de incomodidad para las diferentes partes del cuerpo ${ }^{13}$ consiste en graduar el nivel de incomodidad expresado bajo la forma de dolor en cada parte del cuerpo, en una escala representada por colores, donde: "verde" ningún dolor; "amarillo" dolor soportable que se caracteriza como una leve incomodidad, pero no es incapacitante; "anaranjado" dolor intenso que significa dolor fuerte que obliga al trabajador por algunos momentos a parar la actividad y mantenerse en reposo o cambiar de posición para aliviar el dolor y posteriormente continuar con el trabajo; y "rojo", dolor insoportable que se caracteriza por la incapacidad de continuar con las labores obligando al operario a parar su actividad. La escala divide el cuerpo humano en segmentos y, para cada uno de ellos, se registró el nivel de incomodidad informado en forma subjetiva por cada empleado.

El método RULA (Rapid Upper Limb Assessment) ${ }^{14}$ es un instrumento que permite una evaluación de sobrecarga biomecánica de los miembros superiores y del cuello en una tarea ocupacional. El determinante de riesgo ergonómico en ese método es representado por las posiciones asumidas por los trabajadores en la jornada de trabajo. Las posturas evaluadas son las adoptadas por los miembros superiores, el cuello, tronco y los miembros inferiores. La evaluación del riesgo fue hecha a partir de una observación sistemática de los ciclos de trabajo de los funcionarios puntuando las posturas, frecuencia y fuerza dentro de una escala que varía de 1 (uno), correspondiente al intervalo de movimiento o posturas de trabajo donde la correlación del factor riesgo es mínimo y va hasta el valor 7 (siete), donde el factor de riesgo correlativo es máximo. Para evaluación de los ángulos articulares se utilizó un goniómetro de marca Carci. A partir del análisis de las posturas de los diferentes segmentos, se determinan para cada funcionario cuatro niveles de acción: Nivel 1: la postura es aceptable si no es mantenida o repetida por largos periodos; Nivel 2: son necesarias investigaciones posteriores; algunas intervenciones se pueden volver necesarias; Nivel 3: es necesario investigar y cambiar en breve; $y$, Nivel 4: es necesario investigar y cambiar inmediatamente.

El test utilizado para evaluar la flexibilidad de los músculos isquio-tibiales fue el de Sentarse y alcanzar ${ }^{15}$, siguiendo el estándar canadiense para el test de evaluación de la aptitud física de Canadian Standardized Test of Fitness. ${ }^{16}$ El test fue realizado utilizándose el banco de Wells, midiendo $30,5 \mathrm{~cm}$ x 30,5 x 30,5 $\mathrm{cm}$ con una escala de $26,0 \mathrm{~cm}$ en su prolongación, siendo que el punto cero se encontraba en la extremidad más próxima del evaluado y el $26,0 \mathrm{~cm}$ coincide con el punto de apoyo de los pies. El funcionario retiraba el calzado y en la posición sentada tocaba los pies en la caja con las rodillas extendidas. Con hombros flexionados, codos extendidos y manos sobrepuestas, el trabajador ejecutaba la flexión de tronco al frente debiendo este tocar el punto máximo de la escala con las manos. Fueron realizados tres intentos siendo considerada y establecida la mejor marca.

Los datos fueron analizados por medio de estadística descriptiva (media, desviación estándar, frecuencia relativa) e inferencial. En 
la asociación entre variables categóricas se utilizó el test Chi cuadrado o exacto de Fisher, de acuerdo con las necesidades. En la comparación entre las variables numéricas normales los niveles de comparación. Para las variables numéricas que no tienen normalidad se utilizó el test de U de Mann Whitney o Krusskal Wallis, de acuerdo a los niveles de comparación. La normalidad de los datos fue verificada por medio del test de Shapiro Wilk: se determinó un nivel de significancia al 5\%.

\section{RESULTADOS}

Fueron evaluados 41 funcionarios con edad media de 31,93 años $( \pm 10,165)$, de los cuales $75,6 \%$ mujeres y $24,4 \%$ hombres. Con relación al tiempo de trabajo, la media encontrada en la muestra fue de 5,32 años $( \pm 2,94)$ años.

En la evaluación en la presencia de dolor e incomodidad corporal para las diferentes partes del cuerpo, se pueden observar en la Figura 1 dolores en los hombres $(63,4 \%)$, en la columna cervical $(61,0 \%)$ y en la región de la columna lumbar $(56,1 \%)$ fueron los más frecuentes. No hubo diferencias significativas entre el acontecimiento de estos cuadros y el género de los participantes ( $p>0,05)$.

\section{Gráfico 1.}

Porcentaje de manifestación de dolor e incomodidad por segmento corporal de la muestra.

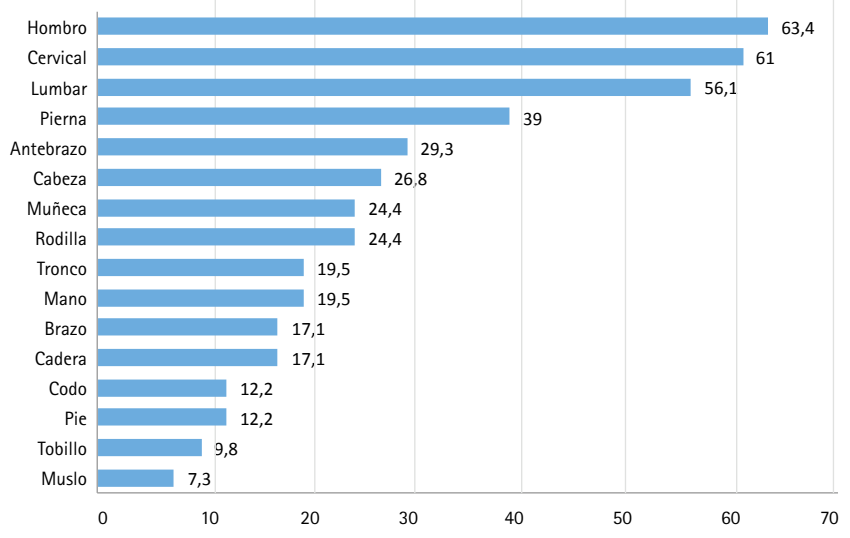

El dolor es uno de los principales sintomas asociados a las disfunciones músculo-esqueléticas relacionadas con el trabajo, de lo cual puede aumentar de forma negativa y evolucionar de forma progresiva en la pérdida de la funcionalidad. ${ }^{17}$ Por lo tanto, la adopción de medidas para el control de esas funciones se vuelven esenciales, tanto en términos sociales como económicos. Para la elaboración de acciones relacionadas con el promedio de la salud del trabajador se hace necesaria la identificación del perfil de los funcionarios y las medidas de los factores de riesgo en el ambiente de trabajo.

Los resultados encontrados en el presente estudio relacionados con el dolor e incomodidad corporal los encontramos en investigaciones con 45 analistas de sistemas de una oficina en la ciudad de Recife, los cuales verifican la continuidad de dolor en las regiones de la comuna lumbar (71\%), seguida por la columna cervical (64\%). De forma semejante, Campos ${ }^{18}$ en un estudio con trabajadores del sector de informática, observó mayor frecuencia de dolor en la columna lumbar $(43,67 \%)$ y hombro $(37,34)$. En este estudio se verificó que todos los trabajadores adoptaban posturas inaceptables conforme RULA; en pocas palabras generaban riesgos a los mismos durante la realización de las tareas laborales. Esos resultados van al encuentro del estudio de Lourinho et $\mathrm{al}^{19}$, sobre riesgos de lesiones músculo-esqueléticas en diferentes sectores de una empresa de calzado, del cual fue verificado que el 78\% de los funcionarios adoptaban posiciones inaceptables, con necesidad de cambios inmediatos y apenas $4 \%$ de los funcionarios adoptaban posiciones con necesidad de intervenciones posteriores. Por lo tanto, Fontes et $\mathrm{al}^{20}$ verificaron en su estudio que los funcionarios que trabajan en la posición sentada presentan menor riesgo postural cuando es comparado con la posición en pie.

\section{DISCUSIÓN}

Para Rumaquella et $\mathrm{al}^{21}$, la postura sentada durante un prolongado tiempo puede llevar a la adopción de una mala postura y, consecuentemente, llevar a la fatiga de los músculos posteriores de soporte de la columna vertebral. Costa e Vieira ${ }^{22}$ resaltan que el aumento de la tensión de los ligamentos de la columna puede causar alteraciones en la presión de los discos intervertebrales finalizando en degeneraciones y, posiblemente, hernia de disco vertebral. La alta frecuencia de manifestaciones de lumbago, también encontrada en el presente estudio, es frecuentemente atribuida a la fatiga y a las deficiencias musculares recurrentes de posturas inadecuadas y repetitivas. Puede ser atribuida a determinadas actividades ocupacionales, principalmente a aquellas en que el trabajador permanece sentado en condiciones antiergonómicas por tiempo prolongado. ${ }^{23}$

Rocha y Debert-Ribeiro ${ }^{24}$, en un estudio con personas que trabajan con análisis de sistemas que desempeñaban sus actividades en la posición sentada, verificaron mayor prevalencia de dolor en columna lumbar (57\%) y cervical/Hombros (55\%) en hombres; en comparación con las mujeres, el predominio fue encontrado en la columna cervical/hombro (74\%) y columna lumbar (72\%). En estudio con 210 funcionarios de una empresa de contratos temporales que proporciona agua y tratamiento de aguas residuales de Bauru que laboraban en actividades sedentarias, se observó predomino de manifestación de dolor principalmente en la columna lumbar $(40,3 \%)$ columna cervical $(27,3 \%)$ y hombros $(23,8 \%){ }^{25}$ En el presente estudio los dolores en los hombros aparecen como una manifestación más prevalentes. En un estudio de revisión, realizado por Mendoza Jr. y Asunción (2005), los autores identificaron que los disturbios del hombro son influenciados por factores biomecánicos relacionados con el trabajo, como flexión o abducción de los hombros por tiempo prolongado, vibraciones, posturas estáticas o con sobrecarga en el miembro superior. Los mismos autores también evidenciaron la asociación entre los disturbios de hombro y los factores psicosociales como estrés, largas jornadas de trabajo y periodo de descanso insatisfactorio.

Ya el dolor cervical viene siendo considerado como uno de los más molestos problemas osteo-musculares, con enorme impacto sobre la salud y calidad de vida de los individuos y en la sociedad como un todo. ${ }^{26}$

La alta prevalencia de manifestaciones de dolor en hombros y en la columna cervical, encontrada en el presente estudio, sugiere la existencia de una relación con una postura adoptada durante la realización de las tareas laborales, recordando que ninguna postura fue considerada aceptable; en pocas palabras, todas las posturas asumidas por los funcionarios generaban riesgo a la salud del trabajador, conforme los criterios del RULA.

De acuerdo con Armijo-Olivo et $\mathrm{al}^{27}$ y Bevilaqua-Grossi, Chaves y 
Oliveira ${ }^{28}$, en las disfunciones cervicales el dolor más frecuente; generalmente está relacionado a los esfuerzos repetitivos y al mantenimiento de posturas inadecuadas durante la actividad ocupacional que acarrean micro-traumatismos a las vértebras cervicales y a los tejidos blandos peri-articulares. De acuerdo con Chaffin y Andersson ${ }^{29}$, la ventana del monitor localizada debajo de la línea de los ojos aumenta la necesidad de flexión cervical y la activación de los músculos extensores del cuello para soportar el peso de la cabeza con la acción de la gravedad.

En cuanto al análisis de las posturas de los diferentes segmentos, utilizándose la herramienta RULA, se observa en la Tabla 1 la frecuencia de los niveles de acción identificados en la muestra del estudio.

Tabla 1.

Frecuencia de los niveles de acción del RULA identificados en la muestra del estudio.

$\begin{array}{lc}\text { Niveles de Acción } & \text { Frecuencia } \\ \text { Nivel } 2 \text { - Algunas intervenciones pueden ser necesarias } & 6 \\ \text { Nivel } 3 \text { - Necesidad de cambio breve } & 20 \\ \text { Nivel } 4 \text { - Necesidad de cambio inmediato } & 15\end{array}$

Se observa que ninguna postura fue considerada aceptable; en pocas palabras, todas las posturas analizadas generan riesgo a la salud del trabajador, siendo que la mayoría necesita de cambios breves $(\mathrm{n}=20)$ o inmediatas $(\mathrm{n}=15)$.

No hubo diferencias significativas entre los niveles de acción del RULA y las variables flexibilidad $(\mathrm{F}=1,803 ; \mathrm{p}=0,179)$ y tiempo de trabajo $(K=2,278 ; \mathrm{p}=0,320)$.

Por lo tanto, se muestran en las Figuras 2 y 3 individuos que necesitan cambio inmediato (Nivel 4 del RULA): presentaron menor flexibilidad y mayor tiempo de trabajo en los demás niveles de acción encontrados en la muestra.

En cuanto a la flexibilidad, la media de la muestra fue de $20,28 \mathrm{~cm}$ $( \pm 10,31)$. En las mujeres la media fue de $20,24 \mathrm{~cm}( \pm 10)$ y en los hombres $20,40 \mathrm{~cm}( \pm 12)$. Se observa en la Tabla 2 que 34 funcionarios tuvieron clasificación bajo la media de los valores normativos, siendo 6 considerado "bajo la media" y 28 considerado "mal".

\section{Figura 1.}

Promedio de flexibilidad de los trabajadores que obtuvieron niveles de acción 2,3 y 4 de puntuación RULA.

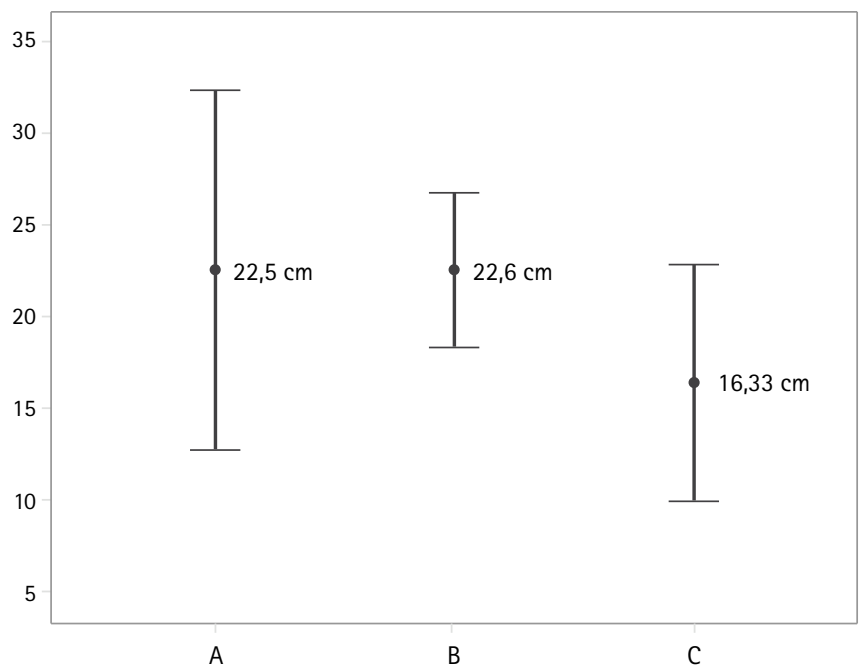

Figura 2.

Promedio del tiempo de trabajo de los funcionarios que obtienen niveles de acción 2, 3 y 4 de puntuación RULA.

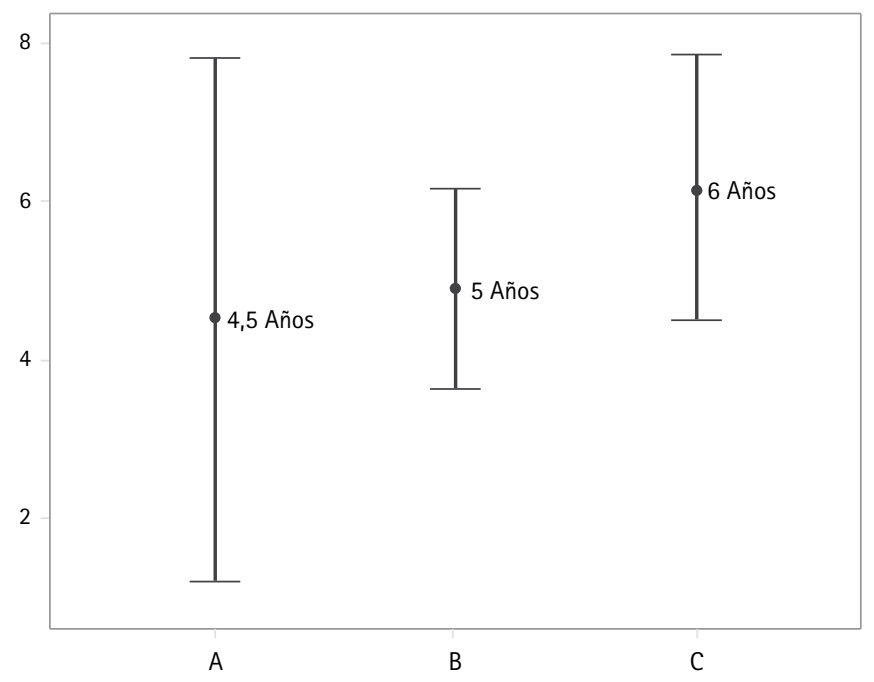

Tabla 2.

Frecuencia de la clasificación del nivel de flexibilidad para el test "sentarse y alcanzar" de la muestra, de acuerdo con el Canadian Standardizes Test of Fitness. ${ }^{16}$

$\begin{array}{lc}\text { Clasificación del nivel de flexibilidad } & \text { Número de funcionarios } \\ \text { Malo } & 28 \\ \text { Abajo de la media } & 6 \\ \text { Media } & 4 \\ \text { Encima de la media } & 3\end{array}$

El lumbago altera la calidad de vida del trabajador, generando limitación funcional y disminución del rendimiento de la ejecución de las tareas laborales, siendo de esta forma considerada una de las principales causas de incapacidad del trabajo, interfiriendo directamente en la calidad de vida de los individuos sintomáticos. ${ }^{30}$

De la misma manera, a la alta frecuencia de dolor e incomodidad corporal encontrado en el presente estudio, parece estar relacionado con los bajos niveles de flexibilidad de la cadena muscular posterior encontrado en la mayoría de la muestra evaluada por el test "Sentarse y alcanzar". Esta posible relación se explica por el hecho de que la flexibilidad al nivel de la columna vertebral y de los músculos isquiotibiales están asociados a la mayoría de las manifestaciones dolorosas en la región lumbar. ${ }^{31}$

La muestra de este estudio presentó alta frecuencia de manifestaciones de dolor e incomodidad corporal (con predominio en hombros, columna cervical y columna lumbar), clasificación del nivel de acción del RULA variando entre 3 y 4 para la mayoría de los participantes en las posturas utilizadas para la realización de la tarea de trabajo, además de bajos niveles de flexibilidad corporal. Estos resultados, separadamente o en conjunto, pueden causar riesgo a la salud del trabajador, afectando su calidad de vida y, posiblemente, interfiriendo en su desempeño y productividad.

\section{CONSIDERACIONES FINALES}

A partir de las condiciones bajo las cuales este estudio fue realizado y considerando los resultados obtenidos, se puede concluir que en la muestra de trabajadores y actividades desarrolladas en la postura 
sentada de una oficina de institución de enseñanza superior, las regiones predominantes indicadas como las manifestaciones de dolor e incomodidad corporal fueron los hombros, la columna cervical y la columna lumbar. En lo que se refiere a la clasificación del nivel de acción del RULA, este varió entre 3 y 4, indicando la necesidad de cambios del proceso de este sistema de producción (principalmente en lo que se refiere a la postura de trabajo). En cuanto a la flexibilidad corporal, los resultados presentan bajos niveles cuando son comparados con los niveles esperados para la edad y género de la muestra. Estos datos apuntan en la necesidad de una nueva disposición ergonómica, viabilizando el aumento de la capacidad productiva y evitando la pérdida en el proceso generado por problemas ergonómicos, además de posibilitar la mejoría de los niveles de salud y seguridad de los trabajadores involucrados.

De esa forma resaltamos la importancia de la investigación, análisis y estudio de los factores de riesgo presentes en los diversos ambientes ocupacionales para posterior planificación e implementación de intervenciones a la promoción y protección de la salud de los trabajadores. Por lo tanto, se sugiere que nuevos estudios sean realizados, con una mayor muestra, viabilizando evidenciar los resultados aquí encontrados, abriendo camino para buscar las soluciones ergonómicas viables, objetivas y eficaces.
1. Saldanha J, Pereira A, Neves R, de Lima M. Facilitadores e barreiras de retorno ao trabalho de trabalhadores acometidos por LER/DORT. Rev bras saúde ocup. 2013; 38(127):122-138,

2. Martins P, Sobrinho C, Silva M, Pereira N, Gonçalves C, Rebouças B, Cartaxo L. Afastamento por doença entre trabalhadores de saúde em um hospital público do estado da Bahia. Rev bras saúde ocup. 2009; 34(120):172-178.

3. Coury H, Moreira R, Dias N. Efetividade do exercício físico em ambiente ocupacional para controle da dor cervical, lombar e do ombro: uma revisão sistemática. Rev bras fisioter (São Carlos). 2009; 13(6):461-479.

4. Armstrong TJ, Buckee $P$, Fine $\amalg$, Hagberg M, Jonsson B, Kilbon A, Kuorinka IA, Silverstein BA, Sjogaard G, Viikari-Juntura ER. A conceptual model for workrelated neck and upper-limb musculoskeletal disorders. Scand J Work Environ Health.1993;19(2):73-84.

5. Diniz $E_{\text {, Carvalho } C M}$, Lemos $A$, Toscano $C F$. Efeitos da ginástica laboral sobre a força de preensão palmar e queixas de dores musculares em auxiliares de produção de uma indústria alimentícia. Fisioter Bras. 2008; 9(5):309-331.

6. Devereux JJ, Vlachonikolis IG, Buckle PW. Epidemiological study to investigate potential interaction between physical and psychosocial factors at work that may increase the risk of symptoms of musculoskeletal disorder of the neck and upper limb. Occup Environ Med. 2002; 59(4):269-77.

7. Kroemer $\mathrm{KH}$, Grandjean E. Manual de ergonomia: adaptando o trabalho ao homen. Porto Alegre: Bookman; 2005.

8. Guimarães BM, Martins LB, De Azevedo LS, Andrade MA. Análise da carga de trabalho de analistas de sistemas e dos distúrbios osteomusculares. Fisioter Mov (Curitiba). 2011; 24(1):115-124.

9. Heyward VH. Avaliação física e prescrição de exercício: técnicas avançadas. Porto Alegre: Artes Médicas; 2004.

10. Resende FD. Análise da distribuição de cargas e das forças entre assento e encosto, na postura sentada adequada [Dissertação Mestrado em Engenharia]. Guaratinguetá: UNESP-Faculdade de Engenharia de Guaratinguetá; 2006. 114 p.

11. Huet, M. Avaliação ergonômica e cinesiológica dos constrangimentos músculoesqueléticos da região sacro-lombar na postura sentada em viagens aéreas longas. [Dissertação Mestrado em Artes e Design]. Rio de Janeiro: Pontifícia Universidade Católica-Departamento de Artes e Design; 2003. 235 p.

12. Serranheira $F$, Pereira $M$, Santos $C S$, Cabrita $M$. Auto referência de sintomas de LME numa grande empresa em Portugal. Rev Port Sau Pub. 2003;21(2):37-48.

13. Moraes LF Os princípios das cadeias musculares na avaliação dos desconfortos corporais e constrangimentos posturais em motoristas do transporte coletivo [Dissertação Mestrado em Engenharia de Produção]. Florianópolis: Universidade Federal de Santa Catarina; 2002. $118 \mathrm{p}$.

14. Mcatamney L, Corlett E. RULA: rapid upper limb assessment: a survey method for the investigation of work-related upper limb disorders. Appl Ergon. 1993; 24(2):91-99.

15. Wells KF, Dillon EK. The sit and reach: a test of back and leg flexibility. Res 0 Exercise Sport. 1952; 23(1):115-118.
16. Canadian Standardized Test of Fitness (CSTF). Operations manual. 3rd ed. Ottawa: Government of Canada, Fitness and Amateur Sport; 1986.

17. Strazdins L, Bammer G. Women, work and musculoskeletal health. Soc Sci Med. 2004; 58(6):997-1005.

18. Campos ES. Distúrbios músculo-esqueléticos em trabalhadores da área de informática: um enfoque à dor lombar. [Dissertação Mestrado em Engenharia de Produção]. Santa Catarina: Universidade Federal de Santa Catarina; $2004.160 \mathrm{p}$.

19. Lourinho MG, Negreiros GR, De Almeida LB, Vieira ER, Quemelo PR. Riscos de lesão musculoesquelética em diferentes setores de uma empresa calçadista. Fisioter Pesq. 2011; 18(3):252-257.

20. Fontes $C F$, Ravagnani IL, Zaia JE, Quemelo PR. Comparação da sobrecarga mecânica em funcionários que executam suas tarefas na posição em pé e sentada. R Bras Ci e Mov. 2013; 21(1):10-15.

21. Rumaquella MR, Menezes MS, Paschoarelli LC, Santos Filho AG. Os efeitos da posição sentada prolongada na coluna vertebral: uma revisão. In: Anais do $8^{\circ}$ Congresso Brasileiro de Desenvolvimento em Design, 8-11 out 2008; Centro Universitário Senac, Sao Paulo. Sao Paulo: AEND Brasil; 2008. p. 4142-4146.

22. Costa BR, Vieira ER. Risk Factors for Work-Related Musculoskeletal Disorders: A Systematic Review of Recent Longitudinal Studies. Am J Ind Med. 2010; 53(3):285-323.

23. Toscano JJ, Egypto EP. A influência do sedentarismo na prevalência de lombalgia. Rev Bras Med Esporte. 2001; 7(4):132-137.

24. Rocha LE, Debert-Ribeiro M. Trabalho, saúde e gênero: estudo comparativo sobre analistas de sistemas. Rev Saúde Pública (São Paulo). 2001;35(6):539547.

25. Vitta A, Canonici AA, Conti MH, Simeao SF. Prevalência e fatores associados à dor musculoesquelética em profissionais de atividades sedentárias. Fisioter Mov. 2012; 25(2):273-280.

26. Kapreli $E_{1}$ Vourazanis $E_{1}$ Billis $E$, Oldham JA, Strimpakos N. Respiratory dysfunction in chronic neck pain patients. A pilot study. Cephalalgia. 2009; 29(7):701-710.

27. Armijo-Olivo $S$, Magee DJ, Parfitt $M$, Major $P$, Thie NM. The association between the cervical spine, the stomatognathic, system and craniofacial pain: a critical review. J Orofac Pain. 2006; 20(4):271-287.

28. Bevilaqua-Grossi D, Chaves TC, Oliveira AS. Cervical Spine signs and symptons: perpetuating rather than predisposing factors for temporomandibular disorder in women. J App Oral Sci (Bauru). 2007;15(4):259-264.

29. Chaffin DB, Andersson GB, Martin BJ. Occupational biomechanics. 4a ed. Hoboken, NJ: Wiley Interscience; 2006.

30. Horng YS, Hwang YH, Wu HC, Liang HW, Mhe YJ, Twu FC, Wang JD. Predicting heal $\neg$ th-related quality of life in patients with low back pain. Spine. 2005; 30(5):551-555.

31. Achour JRA. Flexibilidade. Rev APEF (Londrina). 1994; 9(6)43-52. 